\title{
Identification of Video Game Addiction Using Heart-Rate Variability Parameters
}

\author{
Jung-Yong Kim ${ }^{1}\left(\right.$, Hea-Sol Kim ${ }^{1}\left(\mathbb{D}\right.$, Dong-Joon Kim ${ }^{2, *}$, Sung-Kyun $\operatorname{Im}^{2}{ }^{2}$ and Mi-Sook Kim ${ }^{3}$ \\ 1 Department of HCI, Hanyang University ERICA, Ansan-si 15588, Gyeonggi-do, Korea; \\ jungkim@hanyang.ac.kr (J.-Y.K.); kimheasol@hanyang.ac.kr (H.-S.K.) \\ 2 Department of Industrial and Management Engineering, Hanyang University ERICA, Ansan-si 15588, \\ Gyeonggi-do, Korea; skalon87@gmail.com \\ 3 Department of Clothing and Textiles, Kyung Hee University, Seoul 02447, Korea; mskim@khu.ac.kr \\ * Correspondence: whatsdream@naver.com
}

Citation: Kim, J.-Y.; Kim, H.-S.; Kim, D.-J.; Im, S.-K.; Kim, M.-S. Identification of Video Game Addiction Using Heart-Rate Variability Parameters. Sensors 2021, 21, 4683. https://doi.org/10.3390/ s21144683

Academic Editors: Yvonne Tran and Ki H. Chon

Received: 15 April 2021

Accepted: 28 June 2021

Published: 8 July 2021

Publisher's Note: MDPI stays neutral with regard to jurisdictional claims in published maps and institutional affiliations.

Copyright: (C) 2021 by the authors. Licensee MDPI, Basel, Switzerland. This article is an open access article distributed under the terms and conditions of the Creative Commons Attribution (CC BY) license (https:/ / creativecommons.org/licenses/by/ $4.0 /)$.

\begin{abstract}
The purpose of this study is to determine heart rate variability (HRV) parameters that can quantitatively characterize game addiction by using electrocardiograms (ECGs). 23 subjects were classified into two groups prior to the experiment, 11 game-addicted subjects, and 12 non-addicted subjects, using questionnaires (CIUS and IAT). Various HRV parameters were tested to identify the addicted subject. The subjects played the League of Legends game for 30-40 min. The experimenter measured ECG during the game at various window sizes and specific events. Moreover, correlation and factor analyses were used to find the most effective parameters. A logistic regression equation was formed to calculate the accuracy in diagnosing addicted and non-addicted subjects. The most accurate set of parameters was found to be pNNI20, RMSSD, and LF in the $30 \mathrm{~s}$ after the "being killed" event. The logistic regression analysis provided an accuracy of $69.3 \%$ to $70.3 \%$. AUC values in this study ranged from 0.654 to 0.677 . This study can be noted as an exploratory step in the quantification of game addiction based on the stress response that could be used as an objective diagnostic method in the future.
\end{abstract}

Keywords: HRV parameter; game addiction; League of Legends; stress response; sensitivity; specificity; logistic regression

\section{Introduction}

The game industry is growing, with a market size of more than US $\$ 123.4$ billion worldwide. South Korea is ranked fifth in the world, with $6.7 \%$ of the world market share [1], and accounts for 55.8\% of Korea's content industry exports in 2018 [2]. Ryu and Lee [3] stated that such booming of the game industry has a positive influence on society, including stress management, the realization of the ideal self, and physical ability improvement. In particular, in the current COVID-19 environment, online games are recognized as a complementary means of social distancing [4,5]. However, Internet game players are not protected from becoming addicted to gaming. This addiction problem could adversely affect personal life as well as family and society, and has become a serious public health issue. Byun and Lee [6] found that Internet addiction is closely related to the increased frequency and duration of Internet use, and leads to anxiety, fear, depression, and obsessive-compulsive disorder, with adolescents being vulnerable target users. Koepp et al. [7] observed that dopamine is secreted from the brains of addicted adolescents with a similar pattern to that of drug addiction.

Adverse effects on adolescents have been studied by many authors [8-10]. In particular, it is notable that the most influential factor causing Internet addiction is stress due to excessive competition, and that adolescents exposed to excessive stress sources were readily immersed in the Internet [11]. Adolescents often experience alienation or loneliness when they are addicted to Internet games [12]. They relieve the stress related to daily life 
and loneliness by using internet games, which were easily accessible [13]. The higher the level of stress, the more they tended to fall into game addiction [14]. According to a study by Lee [15], game addiction prevents adolescents from coping with stress sources properly, causing various psychological problems and stress responses. Likewise, the literature indicates that Internet game addiction and mental stress are closely related.

In recent years, heart rate variability (HRV) has been used in many studies to evaluate stress levels [16-19]. Since stress affects the autonomic nervous system (ANS), HRV controlled by the ANS is often referenced as a stress indicator. A number of studies on HRV parameters have been conducted in this regard. Taelman et al. [20] and Vuksanović and Gal [21] observed that the mean of the NN interval, which is often expressed as the RR interval, and the standard deviation of all NN intervals (SDNN) decreased significantly under mental stress. Taelman et al. [20] and Tharion et al. [22] showed that pNN50 (percentage of successive RR intervals greater than $50 \mathrm{~ms}$ ) is significantly decreased under stress. Papousek et al. [23] and Traina et al. [24] reported an increase in the low-frequency power range (LF), a decrease in the high-frequency power range (HF), and a significant increase in the LF/HF ratio when subjects experience stress. Park et al. [25] tested the newly developed measuring system to examine electrocardiograms (ECGs) and found a consistent increase in HR and SDNN as the level of addiction increased. At the same time, the LF and LF/HF parameters showed an obvious increasing trend at a high level of addiction.

On the other hand, Hafeez et al. [26] used EEGs to classify game addicts and nonaddicts using cluster analysis and pattern discrimination. They introduced a statistical method to quantify the addiction phenomenon, and Hafeez et al. [27] and Kim et al. [28] identified the theta and theta/alpha parameters of the right occipital region as the discriminating variables between addicts and non-addicts. Likewise, the attempt to quantify the particular characteristics of addiction is an ongoing topic for researchers. If such a numerically quantifiable approach can be successful and assist physicians in identifying an addicted patient, they will be able to treat the patient more efficiently and objectively. Therefore, in this study, the authors are challenged to search for a quantifiable indicator of addiction in ECG response by investigating various HRV parameters. The purpose of this study is to extract quantitative HRV parameters that characterize the particular stress response of game addicts. To achieve this research goal, an exhaustive approach was performed by testing all the candidate parameters collected using window sizes of 30, 60, 90, and $120 \mathrm{~s}$.

\section{Methods}

\subsection{Subjects}

A total of 23 male students participated in the experiment. The mean age was 23 years ( \pm 3 years). Eleven participants were addicted, and 12 of them were non-addicted. They were categorized using the Compulsive Internet Use Scale (CIUS) by Meerkerk et al. [29], and the Internet Addiction Test (IAT) by Young and De Abreu [30]. Based on CIUS, subjects with 2.5 or higher were categorized as addicted, and those with scores less than 1.5 were categorized as non-addicted [31]. An IAT score of 50 or higher has been used to classify the game-addicted by many researchers [10,32-35]. In this study, a subject was categorized as a game addict only when the subject met both the IAT and CIUS standards. For nonaddicted subjects, an IAT score of 40 or lower was required. 14 addicted subjects and 14 non-addicted subjects were selected. 3 addicted subjects and 2 non-addicted subjects were discarded due to a technical error in the measurement system. Controlling the compounding effect of gender in this study, only male participants were tested in this study. Alcohol consumption was prohibited for $24 \mathrm{~h}$ before the start of the experiment, and smoking and coffee consumption were prohibited for $1 \mathrm{~h}$ before the start of the experiment. A fee was paid to the participants. The experiment was conducted in accordance with the regulations under consideration by the Institutional Review Board of Hanyang University in the Republic of Korea (IRB approval number: HYU-2019-08-004-1). 


\subsection{Apparatus}

The questionnaires used to categorize subjects into two groups prior to the experiment were the CIUS by Meerkerk et al. [29] and the IAT by Young and De Abreu [30,36].

League of Legends by Riot Games Inc. (Los Angeles, CA, USA) was chosen for the experiment. This game was one of the most frequently played games among internet game players [37], and the frequent battles in the game made players experience a simulated life and death situation associated with probable stress reactions.

For data collection, an auxiliary channel of QEEG-64FX by LAXTHA Inc. (Daejeon, Republic of Korea) was used for ECG measurements (Figure 1). A data collection program called Telescan was used. The data sampling rate was set to $500 \mathrm{~Hz}$. The experiment was conducted in a room equipped with a computer, a table, and a chair, where other external stimuli were restrained.

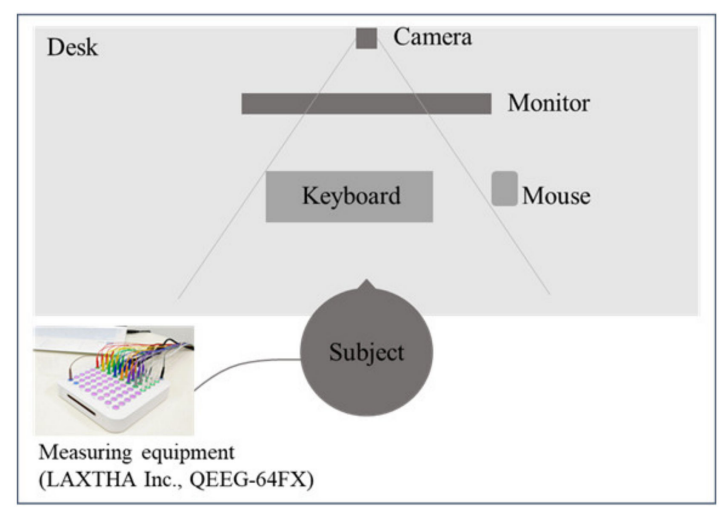

(a)

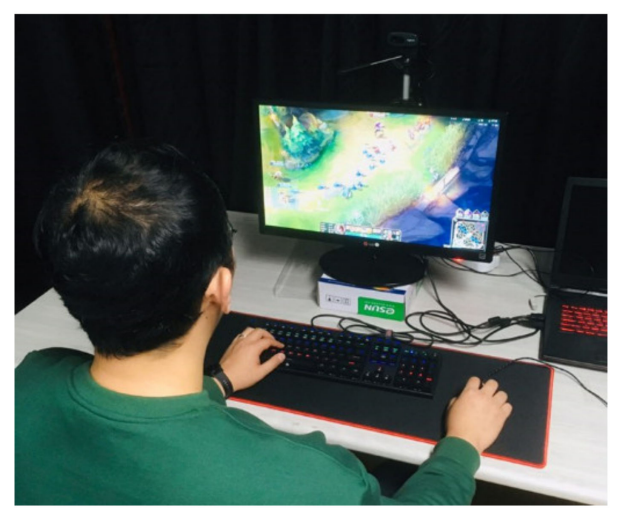

(b)

Figure 1. ECG measurement equipment. (a) Top view of experimental set-up, (b) The experimental scene.

\subsection{Experimental Design}

The experiment was designed to test HRV parameters to determine whether they could differentiate subjects into two groups: addicted and non-addicted. A betweensubjects design was used in this study. The independent variables were the addiction status of the group, and the dependent variables were 14 parameters, including 7 time-domain variables and 7 frequency-domain variables. The time-domain parameters are $\mathrm{NN}$ interval average (RR interval average), SDNN, SDSD, pNNI50, pNNI20, RMSSD, and heart rate average (Table 1). The frequency-domain parameters are LF, HF, LF/HF ratio, LFnu, HFnu, total power, and VLF (Table 2). This study observed specific events during gameplay, including a "killed event", when a player's character was killed by an opponent, and a "killing event", when the player killed an opponent. The data collection window sizes for these events were $30 \mathrm{~s}, 60 \mathrm{~s}, 90 \mathrm{~s}$, and $120 \mathrm{~s}$, respectively, to consider the possible delay of the response.

\subsection{Procedure}

Positive electrode was placed in the V1 location (between the right rib 3 and 4), and the negative electrode was placed in the left infraclavicular fossa according to the standard limb guidance method [39]. The experimental procedure was briefly explained to the subject, and the ECG sensors were attached and tested to ensure that stable signals were obtained for $1 \mathrm{~min}$ while the subjects were relaxing. A "normal game", which is a practice game that does not affect the player's score, was played for familiarization; a "ranked game", which is a competing game affecting the player's score, was played for 30-40 min. For players' immersion in the game, the ranked game was played based on the individual skill level. Subjects played a "normal game" once and a "ranked game" twice, while the ECG was obtained. Subjects were not informed about the addiction test score; thus, they 
did not know whether they were categorized in the addicted group or not. The detailed experimental procedure is shown in Figure 2.

Table 1. Time-domain variables for heart rate variability [38].

\begin{tabular}{|c|c|c|}
\hline Variable & Description & Equation \\
\hline Mean NNI & Mean NN intervals & $\frac{1}{N} \sum_{i=1}^{N} R R_{i}$ \\
\hline SDNN & Standard deviation of all NN intervals & $\sqrt{\frac{1}{N} \sum_{i=1}^{N}(R R i-\overline{R R})}$ \\
\hline SDSD & Standard deviation of differences between adjacent NN intervals & $\sqrt{\frac{1}{N-1} \sum_{i=1}^{N-1}\left(\left|R R_{i}-R R_{i+1}\right|-\overline{R R d i f}\right)^{2}}$ \\
\hline pNNI50 & pNN50 count divided by the total number of all NN intervals (\%) & $\frac{\sum_{i=1}^{M}\left\{\left|R R_{i+1}-R R_{i}\right|>50 \mathrm{~ms}\right\}}{N} \times 100$ \\
\hline pNNI20 & pNN20 count divided by the total number of all NN intervals (\%) & $\frac{\sum_{i=1}^{M}\left\{\left|R R_{i+1}-R R_{i}\right|>200 \mathrm{~ms}\right\}}{N} \times 100$ \\
\hline RMSSD & $\begin{array}{l}\text { The square root of the mean of the sum of the squares of } \\
\text { differences between adjacent NN intervals }\end{array}$ & $\sqrt{\frac{1}{N-1} \sum_{i=1}^{N-1}\left(R R_{i+1}-R R_{i}\right)^{2}}$ \\
\hline Mean HR & Mean heart rate & $\frac{1}{N} \sum_{i=1}^{N} H R_{i}$ \\
\hline
\end{tabular}

Table 2. Frequency-domain variables of heart rate variability [38].

\begin{tabular}{ccc}
\hline Variable & Description & Frequency Range \\
\hline LF & Power in low-frequency range & $0.04-0.15 \mathrm{~Hz}$ \\
HF & Power in high-frequency range & $0.15-0.4 \mathrm{~Hz}$ \\
LF/HF ratio & Sympathovagal balance & \\
LFnu & LF power in normalized units: (LF/(total power - VLF) $) \times 100$ & \\
HFnu & HF power in normalized units: (HF/(total power - VLF) $\times 100$ & Approximately $\leq 0.4 \mathrm{~Hz}$ \\
Total Power & The variance of NN intervals over the temporal segment & $\leq 0.04 \mathrm{~Hz}$ \\
VLF & Power in very low-frequency range & \\
\hline
\end{tabular}

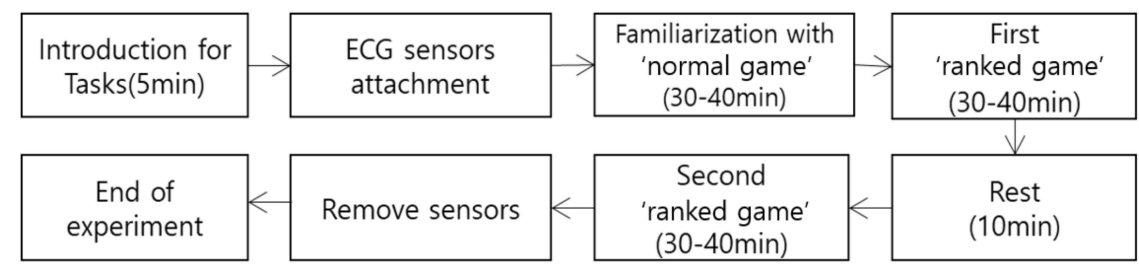

Figure 2. Experimental process.

\subsection{Data Analysis}

The data were analyzed in batches using Python, and time series analysis and frequency analysis were performed at the same time. The parameters used for time series analysis were extracted by using the Christov ECG R-peak segmentation algorithm. The extracted parameters were NN interval average, SDNN, RMSSD, pNNI50, pNNI20, SDSD, and heart rate average. The signal was also extracted and transformed into frequency parameters using the fast Fourier transform. Welch's periodogram was applied to estimate the spectral properties of the HRV signals, using a Hanning window. VLF (power in verylow-frequency ranges, 0.0033-0.04 Hz), LF (power in low-frequency ranges, 0.04-0.15 Hz), HF (Power in high-frequency ranges, $0.15-0.4 \mathrm{~Hz}$ ), and total power (Power in all the frequency ranges, $\leq 0.4$ ) were obtained by the sum of the power in the relevant frequency range of the spectrum. Based on these power values, the values of LF/HF ratio, LFnu, and HFnu were calculated. 
Normality was tested by using Kolmogorov-Smirnov test for individual data set. The dataset with a low normality value was graphically examined to ensure an adequate level of normality. During the process, illegal outliers were treated. The $t$-test was performed $(p<0.1)$ to find the parameters and window size that statistically differentiate two groups: the addicted and non-addicted. The statistical analysis was an exhaustive process used to identify the set of most effective parameters and the window size. A correlation analysis was also performed to determine the redundancy of parameters, and a factor analysis was performed to choose the main parameters representing the characteristics of each group. Finally, a logistic regression analysis was conducted to test the sensitivity and specificity of the statistical model in identifying addicted or non-addicted subjects based on the current experimental data. The analysis process is illustrated in Figure 3. Statistical analysis was performed using SPSS Statistics 24.

\begin{tabular}{|c|}
\hline $\begin{array}{c}\text { t-test for all parameters } \\
\text { at all window sizes and events }\end{array}$ \\
\hline $\begin{array}{c}\text { Correlation analysis and Factor analysis } \\
\text { to sort out the parameters minimizing } \\
\text { redundancy and better representing } \\
\text { the attribute of parameters }\end{array}$ \\
\hline \multicolumn{2}{|c|}{ Sensitivity testing } \\
for selecting the parameters \\
\hline Parameters for future diagnostic study \\
\hline
\end{tabular}

Figure 3. Data analysis process.

\section{Results}

An elimination process was used to sort out the best combination of parameters out of 14 parameters from 4 window sizes through statistical analyses.

\subsection{The t-Test Results between Groups by Window Size}

There were no significant differences in average parameter values between the addicted and non-addicted groups for the entire window sizes during the experiment $(p>0.1)$.

\subsection{The t-Test Results between Groups after Specific Event}

There was no significant difference of HRV parameters between groups for window sizes of $30 \mathrm{~s}, 60 \mathrm{~s}, 90 \mathrm{~s}$, and $120 \mathrm{~s}$ after "killing events" ( $p>0.1)$. However, as shown in Tables 3 and 4, the HRV parameters measured for window sizes of $30 \mathrm{~s}$ and $60 \mathrm{~s}$ after "killed events" showed a significant difference in some parameters between the two groups. In particular, pNNI20 and LF showed a significant difference $(p<0.05)$, and a marginally significant difference was observed for SDSD, RMSSD, and total power $(p<0.1)$.

\subsection{Correlation Analysis and Factor Analysis with HRV Parameters}

A correlation analysis was performed to examine the redundancy of the parameters in differentiating between the two groups. LF and pNNI20 with significant $p$-values $(p<0.05)$ in the $t$-test indicated a low correlation coefficient (0.264). Both could be used to improve statistical power in differentiating the two groups. On the other hand, SDSD and RMSSD showed a correlation coefficient of 1.000, and the total power and LF indicated a coefficient of 0.958 . Thus, only one parameter was used to build the statistical model. Therefore, the correlation analysis suggested that the combination of the [pNNI20, LF, SDSD] or [pNNI20, LF, RMSSD] parameter set could be the best combination of parameters with the least redundancy. 
Factor analysis was also performed to examine whether the selected parameters covered various factors of the data (Figure 4). The parameters with high eigenvalues for Factor 1 were RMSSD, SDSD, pNNI_50, and pNNI_20, and the parameters with high eigenvalues for Factor 2 were LF, total power, and SDNN. That is, the [pNNI20, LF, SDSD] or [pNNI20, LF, RMSSD] parameter set from the correlation analysis (Table 5) were found to have the highest eigenvalues for both Factor 1 and Factor 2 (Table 6). Therefore, the final combination of parameters for statistical modeling was [pNNI20, LF, RMSSD] or [pNNI20, LF, SDSD]. In logistic regression modeling, [pNNI20, LF, RMSSD] was arbitrarily selected to test the model performance in this study because both RMSSD and SDSD were highly correlated with each other $(r=1.000)$.

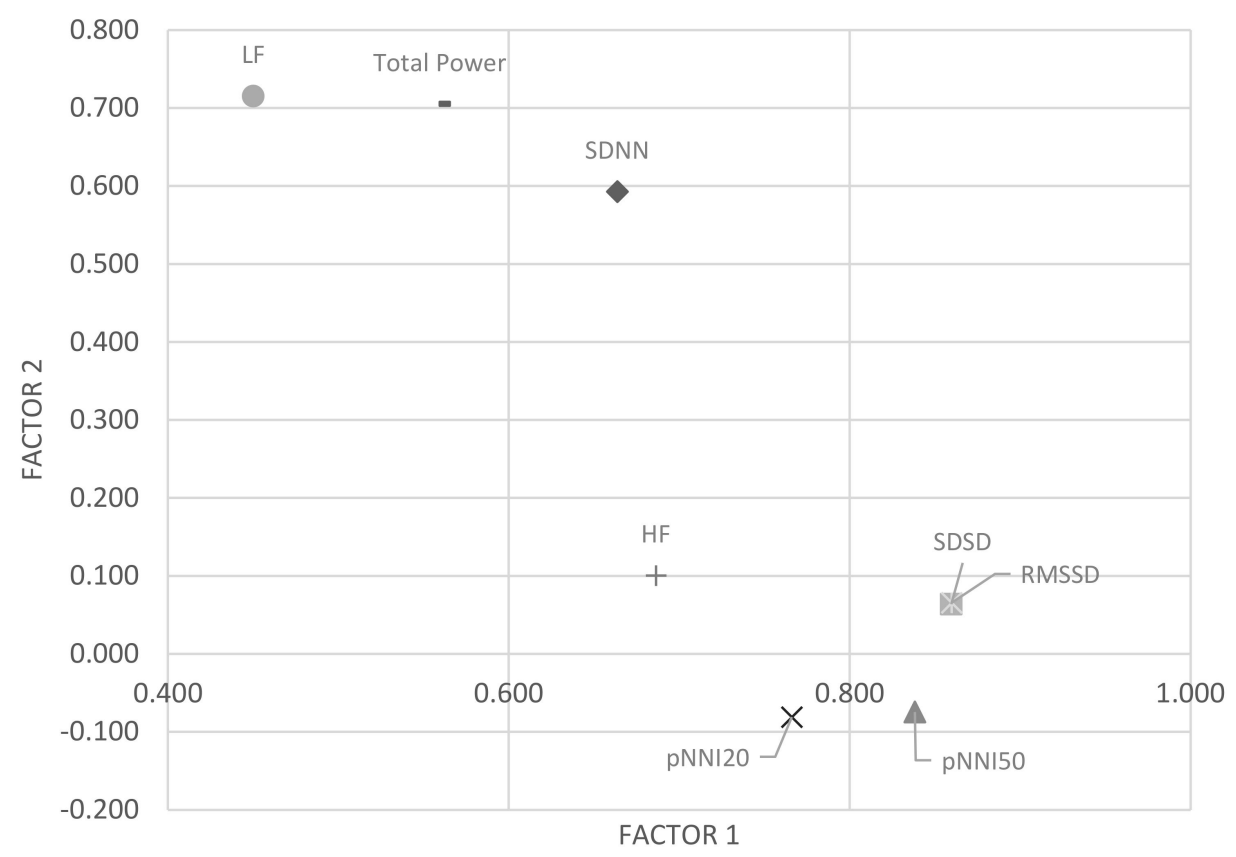

Figure 4. Factor analysis results.

Table 3. The $t$-test results for data from $30 \mathrm{~s}$ window size after "killed event"; mean ( \pm standard deviation).

\begin{tabular}{cccc}
\hline Parameter & Addicted Group & Non-Addicted Group & $p$-Value \\
\hline MeanNNI & $697.72( \pm 81.42)$ & $672.16( \pm 82.23)$ & 0.134 \\
SDNN & $45.31( \pm 14.52)$ & $41.2( \pm 14.53)$ & 0.174 \\
SDSD & $28( \pm 14.05)$ & $23.35( \pm 12.77)$ & $* 0.093$ \\
pNNI50 & $7.15( \pm 9.41)$ & $5.21( \pm 10.9)$ & 0.368 \\
pNNI20 & $37.53( \pm 16.67)$ & $29.15( \pm 17)$ & $* 0.018$ \\
RMSSD & $28.1( \pm 14.04)$ & $23.45( \pm 12.78)$ & $* 0.093$ \\
MeanHR & $87.5( \pm 9.86)$ & $90.91( \pm 10.72)$ & 0.116 \\
LF & $1009.35( \pm 798.74)$ & $679.67( \pm 596.46)$ & $* 0.020$ \\
HF & $326.26( \pm 312.39)$ & $230.59( \pm 312.38)$ & 0.141 \\
LF/HF ratio & $5.16( \pm 6.8)$ & $6.45( \pm 10.06)$ & 0.489 \\
LFnu & $71.93( \pm 19.13)$ & $72.41( \pm 19.2)$ & 0.904 \\
HFnu & $28.07( \pm 19.13)$ & $27.59( \pm 19.2)$ & 0.904 \\
Total Power & $1640.71( \pm 1084.45)$ & $1260.54( \pm 970.6)$ & $* 0.072$ \\
\hline$* *<0.05 * p<0.1$. & & &
\end{tabular}


Table 4. The $t$-test results for data from $60 \mathrm{~s}$ window size after "killed event"; mean ( \pm standard deviation).

\begin{tabular}{cccc}
\hline Parameter & Addicted Group & Non-Addicted Group & $p$-Value \\
\hline MeanNNI & $714.36( \pm 76.27)$ & $690.67( \pm 80.38)$ & 0.154 \\
SDNN & $53.97( \pm 23.08)$ & $50.21( \pm 18.52)$ & 0.380 \\
SDSD & $29.24( \pm 13.76)$ & $28.96( \pm 21.42)$ & 0.943 \\
pNNI50 & $7.84( \pm 9.18)$ & $7.14( \pm 12.55)$ & 0.768 \\
pNNI20 & $37.88( \pm 16.86)$ & $32.56( \pm 17.04)$ & 0.137 \\
RMSSD & $29.29( \pm 13.78)$ & $28.98( \pm 21.42)$ & 0.939 \\
MeanHR & $85.52( \pm 9.35)$ & $88.8( \pm 10.15)$ & 0.115 \\
LF & $973.06( \pm 630.72)$ & $753.93( \pm 536.36)$ & $* 0.071$ \\
HF & $303.23( \pm 268.49)$ & $315.82( \pm 459.75)$ & 0.880 \\
LF/HF ratio & $4.21( \pm 2.75)$ & $4.51( \pm 4.3)$ & 0.708 \\
LFnu & $77.15( \pm 8.37)$ & $74.02( \pm 14.85)$ & 0.244 \\
HFnu & $22.85( \pm 8.37)$ & $25.98( \pm 14.85)$ & 0.244 \\
Total Power & $1781.33( \pm 1129.5)$ & $1672.64( \pm 1251.71)$ & 0.668 \\
VLF & $505.03( \pm 441.69)$ & $602.89( \pm 539.92)$ & 0.356
\end{tabular}

Table 5. Correlation coefficients among heart rate variability parameters.

\begin{tabular}{ccccccccc}
\hline & SDNN & SDSD & pNNI50 & pNNI20 & RMSSD & LF & HF & $\begin{array}{c}\text { TOTAL } \\
\text { POWER }\end{array}$ \\
\hline SDNN & & & & & & & \\
SDSD & $0.684^{* *}$ & & & & & & \\
pNNI50 & $0.477^{* *}$ & $0.639^{* *}$ & & & & & \\
pNNI20 & $0.405^{* *}$ & $0.480^{* *}$ & $0.797^{* *}$ & & & & \\
RMSSD & $0.686^{* *}$ & $1.000^{* *}$ & $0.639^{* *}$ & $0.480^{* *}$ & & & \\
LF & $0.614^{* *}$ & $0.337^{* *}$ & $0.265^{* *}$ & $0.264^{* *}$ & $0.337^{* *}$ & & \\
HF & $0.483^{* *}$ & $0.608^{* *}$ & $0.402^{* *}$ & $0.267^{* *}$ & $0.607^{* *}$ & $0.545^{* *}$ & \\
Total & $0.731^{* *}$ & $0.460^{* *}$ & $0.320^{* *}$ & $0.275^{* *}$ & $0.461^{* *}$ & $0.958^{* *}$ & $0.680^{* *}$ & \\
Power & & & & & & & \\
** Correlation is significant at the 0.01 level (both sides).
\end{tabular}

Table 6. The eigenvalues from factor analysis.

\begin{tabular}{ccc}
\hline & Factor $\mathbf{1}$ & Factor $\mathbf{2}$ \\
\hline SDNN & 0.664 & 0.593 \\
SDSD & 0.860 & 0.064 \\
pNNI50 & 0.838 & -0.075 \\
pNNI20 & 0.766 & -0.081 \\
RMSSD & 0.860 & 0.066 \\
LF & 0.450 & 0.715 \\
HF & 0.686 & 0.101 \\
Total Power & 0.559 & 0.705 \\
\hline
\end{tabular}

\subsection{Logistic Regression Models}

Logistic regression models were developed using the selected parameters. A total of 15 mathematical equations were designed to test the maximum sensitivity and specificity of the parameters using natural logarithms and squares. In terms of identifying the addicted group, the sensitivity was computed, and ranged from 0.324 to 0.400 ; the specificity ranged from 0.828 to 0.922 . The overall accuracy ranged from $67.7 \%$ to $70.3 \%$. The model with the highest specificity of 0.922 was constructed using pNNI20, $\ln ($ RMSSD), and LF. The model with the highest sensitivity of 0.400 was obtained using $\ln (\mathrm{pNNI} 20),(\mathrm{RMSSD})^{2}$, and $\ln (\mathrm{LF})$. The model with the highest overall accuracy of $70.3 \%$ was obtained using pNNI20, $\ln ($ RMSSD), and LF. The second-highest overall accuracy model (69.7\%) was obtained using $\ln (\mathrm{pNNI} 20), \ln (\mathrm{RMSSD})$, and $(\mathrm{LF})^{2}$. The results are summarized in Table 7. 
Table 7. Summary results of four logistic regression models with the highest accuracy.

\begin{tabular}{|c|c|c|c|c|c|}
\hline Model No. & Parameter & Model Equation & Sensitivity & Specificity & Accuracy (\%) \\
\hline Model 1 & $\begin{array}{l}\text { pNNI20 } \\
\text { RMSSD } \\
\text { LF }\end{array}$ & $\begin{array}{c}1 /(1+\exp (-(-1.705+0.048 \times \mathrm{pNNI} 20- \\
0.035 \times \mathrm{RMSSD}+0.0005 \times \mathrm{LF})))\end{array}$ & 0.324 & 0.906 & 69.3 \\
\hline Model 2 & $\begin{array}{c}\ln (\mathrm{pNNI} 20) \\
(\mathrm{RMSSD}) 2 \\
\ln (\mathrm{LF})\end{array}$ & $\begin{array}{l}1 /(1+\exp (-(-9.911+2.309 \times \ln (\mathrm{pNNI} 20) \\
\left.\left.\left.\quad+0.0003 \times(\mathrm{RMSSD})^{2}+0.272 \times \ln (\mathrm{LF})\right)\right)\right)\end{array}$ & 0.400 & 0.828 & 67.7 \\
\hline Model 3 & $\begin{array}{c}\mathrm{pNNI20} \\
\ln (\mathrm{RMSSD}) \\
\text { LF }\end{array}$ & $\begin{array}{c}1 /(1+\exp (-(1.232+0.054 \times \mathrm{pNNI} 20- \\
1.292 \times \ln (\text { RMSSD })+0.0006 \times \mathrm{LF})))\end{array}$ & 0.324 & 0.922 & 70.3 \\
\hline Model 4 & $\begin{array}{c}\ln \left(\mathrm{pNNI}^{20}\right) \\
\ln (\mathrm{RMSSD}) \\
(\mathrm{LF}) 2\end{array}$ & $\begin{array}{c}1 /(1+\exp (-(-5.5417+2.505 \times \ln (\mathrm{pNNI} 20)- \\
\left.\left.\left.1.271 \times \ln (\mathrm{RMSSD})+0.0000002 \times \mathrm{LF}^{2}\right)\right)\right)\end{array}$ & 0.891 & 0.343 & 69.7 \\
\hline
\end{tabular}

\subsection{Characteristics of Distributions Affecting the Sensitivity and Specificity}

The true positive rate (sensitivity) was less than 0.4 in the above analysis, which is not good enough to provide a diagnosis of addiction for medical treatment. Such a relatively low sensitivity could be a part of the outcome based on the logistic regression model to maximize the total accuracy. To see the characteristics of the probability distribution of the data, Figures 5-7 are shown under the assumption of a normal distribution. As shown, there is a substantial overlap between distributions that could make either sensitivity or specificity low. From the observations, the criterion beta used for decision-making seemed to be biased to a conservative standard rather than a liberal one, considering that the specificity was much higher than the sensitivity. For example, for Model 1, with a maximum accuracy of $72.3 \%$, the cut-off point associated beta value was set to 0.523 , and the sensitivity and specificity were computed as 0.324 and 0.953 , respectively. If a different cutoff value was then used, such as 0.372 in Model 2, the sensitivity and specificity can be computed as 0.656 and 0.703 , respectively, with $67.3 \%$ accuracy.

\subsection{Area under the Curve (AUC) Values}

Figure 8 shows the ROC curves of the four models. The AUC value of 0.677 was for Model 1, 0.655 for Model 2, 0.673 for Model 3, and 0.654 for Model 4. According to Hosmer and Lemeshow's study [40], models having an AUC value of 0.5 or less have no discriminating power. A model can be considered acceptable only if the AUC value is between 0.7 and 0.8 , and a model has excellent discriminating power if the AUC value is between 0.8 and 0.9 . Thus, the AUC value of the current logistic regression model is close to the acceptable level, but further refinement is required for the model to be acceptable.

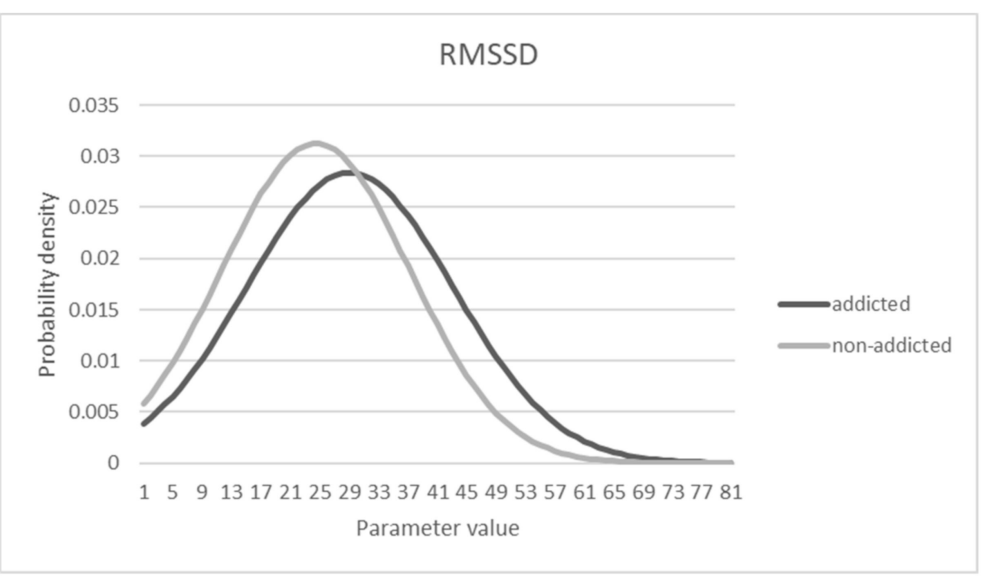

Figure 5. Probability density distribution of RMSSD parameter. 


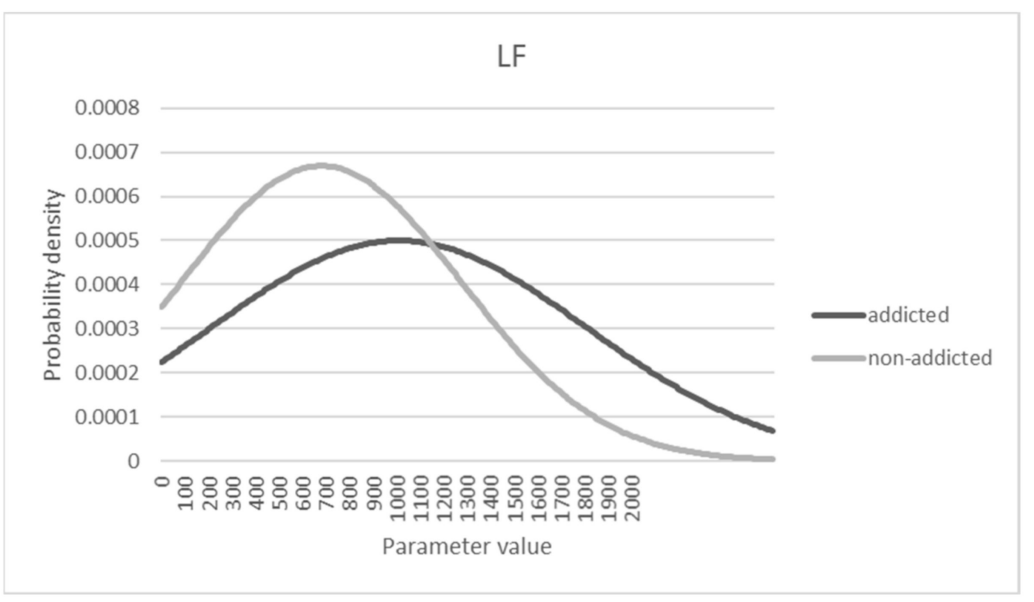

Figure 6. Probability density distribution of LF parameter.

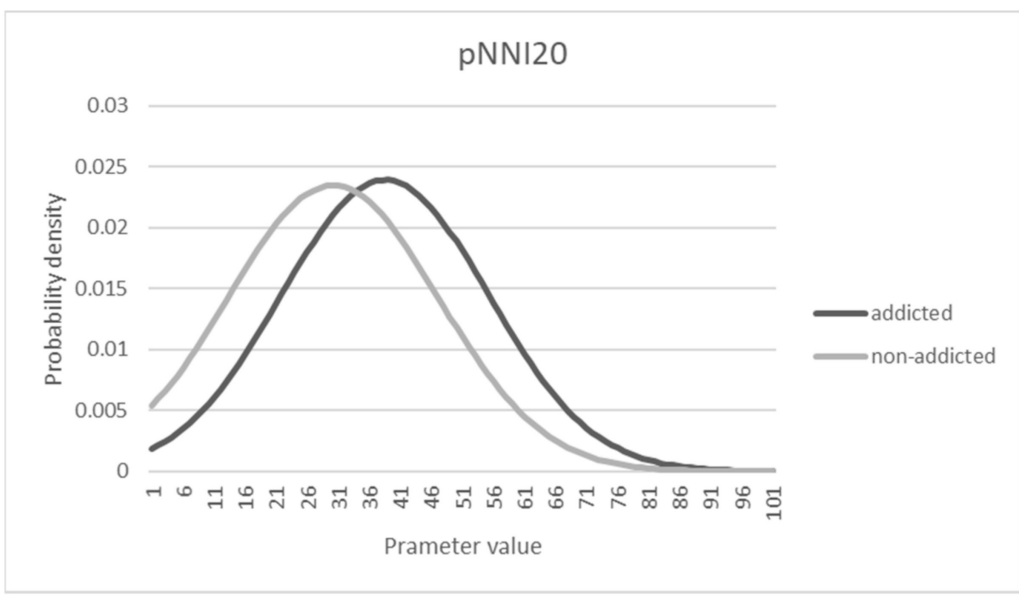

Figure 7. Probability density distribution of pNNI20 parameter.

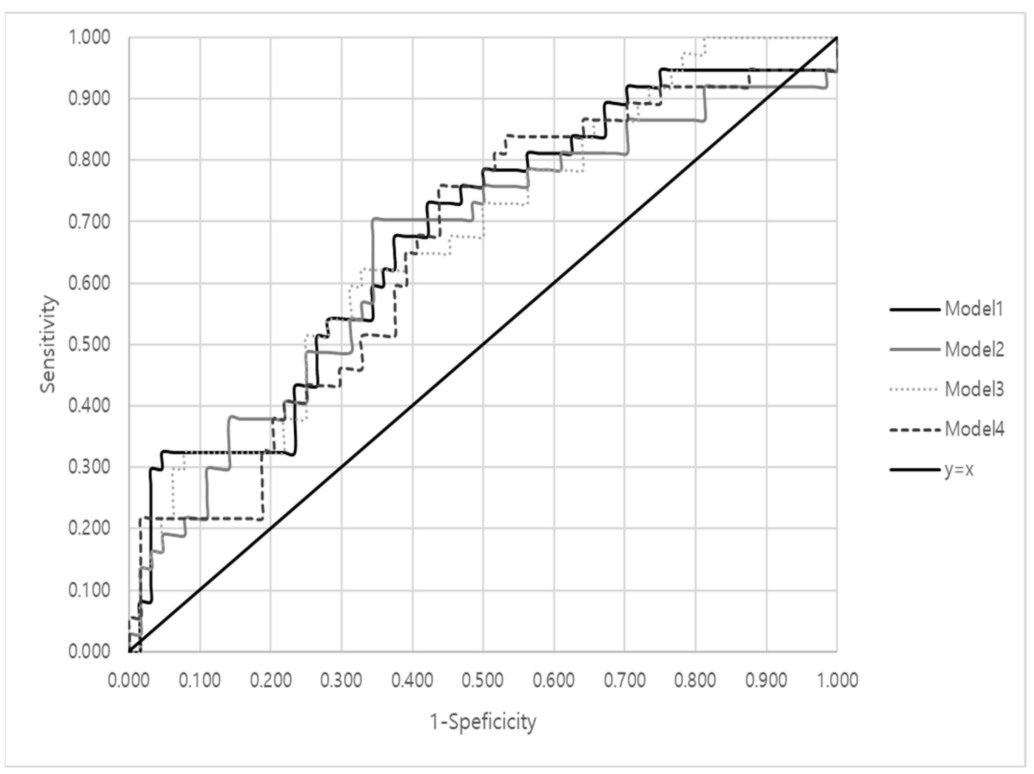

Figure 8. Receiver operating characteristic (ROC) curves of four representative models. 


\section{Discussion}

The study showed that "being killed" in a virtual situation generated a greater signal response among the addicted subjects than non-addicted subjects. Klimt et al. [41] mentioned that a shift in self-perception would occur while enjoying the game and identifying oneself with the game character or when playing games experiencing flow or psychological mastery. Turkay and Kinzer [42] stated that the customization process of avatars by players could greatly influence players to identify themselves as game characters. Therefore, it is reasonable to think that such an affective attachment with an avatar could psychologically influence the players, and this phenomenon could be even more severe among addicted subjects than non-addicted ones.

Regarding the model building, three different statistical methods were used to select the parameters to build the best logistic regression model. Through the $t$-test, the pNNI20 and LF parameters were selected because they showed the most significant results $(p<0.05)$ in differentiating the two groups $30 \mathrm{~s}$ after the "being killed" event. This means that both time-domain and frequency-domain parameters could be effective in statistically discriminating between the two groups. The total power parameter showed a significant $p$-value $(<0.072)$; however, it was not selected for the final logistic model because it was highly correlated with the LF parameter $(\mathrm{r}=0.958)$ to avoid redundancy. In addition, the RMSSD (or SDSD) parameter was used for the logistic regression model because it showed the highest eigenvalue (0.86) of Factor 1 in the factor analysis. The LF parameter with a significant $p$-value in the $t$-test also showed the highest eigenvalue (0.715) for Factor 2, which was used for the final logistic regression model.

The final parameters selected in this study were found to be associated with the stress response based on previous studies. Bernardi et al. [43] evaluated HRV parameters under the mentally stressful situation of a subject performing arithmetic while speaking or reading, and they observed the increased power of LF when subjects were hurrying to perform the calculation task. Huang et al. [44] found that RMSSD and the combination of various variables had a positive correlation with mental fatigue induced by mental stress. According to a study by Jang et al. [45], RMSSD was also found to have a marginal correlation with tension $(\mathrm{r}=0.268, p=0.039)$, depression $(\mathrm{r}=0.356, p=0.005)$, fatigue $(\mathrm{r}=0.259, p=0.041)$, and frustration $(\mathrm{r}=0.304, p=0.018)$. Lee et al. [46] observed changes in HRV during physical and mental stress in patients with depression, and they reported a significant increase in RMSSD during the stress period compared with the rest period. Mallinani et al. [47] explained that increased sympathetic activity could be functionally characterized by an increase in the LF component in terms of LF-HF balance. Kim et al. [48] reviewed the function of HRV parameters and concluded that low parasympathetic activity was frequently related to a decrease in HF and an increase in LF.

To investigate the efficacy of the regression model in diagnosing game addiction patients, the AUC values were calculated and compared with the reference values. The computed AUC value in this study ranged from 0.654 to 0.677 , which is known to have insufficient accuracy for field applications. This indicated that the increased stress response of the addicted during a "killed event" was statistically meaningful, but it might not fully reflect the symptom of addiction that the players were experiencing. Regarding the sensitivity and specificity score, the sensitivity was computed and ranged from 0.324 to 0.400 , and the specificity ranged from 0.828 to 0.922 based on the logistic regression model with the default cut-off point used as a decision criterion. However, the values could change when different cut-off points were used. For now, the AUC value was less than 0.7, which could expect only less-than-accurate decision-making. Therefore, it is necessary to test the model performance under various experimental conditions. At any rate, it is important to understand the nature of HRV parameters among addicted game players, who have been very responsive to stressful stimuli, which was worthwhile to investigate further for quantification of addictive symptoms during game playing. 


\section{Conclusions}

In this study, the difference in HRV parameters between the addicted and non-addicted group was measured during game playing, and it was found that pNNI20, RMSSD, and LF reflected the difference in stress response sensitively for a window size of $30 \mathrm{~s}$ after a "being killed" event. To identify the difference between the game-addicted and non-addicted subjects, the AUC score was computed and found to be less than accurate. The quantification of the psychophysiological response of the addictive game was a challenging task, as was shown in this study, but it is worth pursuing the prevention and rehabilitation of addicted patients in the future. For further study, various types and greater numbers of subjects need to be tested for better representation of the addiction symptoms. Additional mathematical exploration using artificial intelligence techniques could be another option for analyzing bio-information with a high level of variability and probable irregularity. It would also be intriguing to examine and compare the HRV parameters to other psychophysiological signals to identify the unknown patterns of game addiction.

Author Contributions: J.-Y.K., H.-S.K., D.-J.K., S.-K.I. and M.-S.K. drafted parts of the manuscript and reviewed and edited the full manuscript. All authors have read and agreed to the published version of the manuscript.

Funding: This research was funded by the Ministry of Education and the Ministry of Science and ICT, grant numbers No.NRF-2018R1D1A1B07050786 and No. 2020-0-01343.

Institutional Review Board Statement: This experiment was conducted in accordance with the regulations under consideration by the Institutional Review Board of Hanyang University in the Republic of Korea (IRB approval number: HYU-2019-08-004-1).

Informed Consent Statement: Informed consent was obtained from all subjects involved in the study. Data Availability Statement: Not applicable.

Acknowledgments: This research was partly supported by a National Research Foundation of Korea (NRF) grant funded by the Ministry of Education (No.NRF-2018R1D1A1B07050786, Development of the algorithm to identify the EEG pattern of game addicts) and the Institute of Information \& Communications Technology Planning \& Evaluation (IITP) grant funded by the Korean government (MSIT) (No. 2020-0-01343, Artificial Intelligence Convergence Research Center (Hanyang University ERICA)).

Conflicts of Interest: The authors declare no conflict of interest.

\section{References}

1. Korea Creative Content Agency. Accounts Settlement and 2018 Issue Analysis; Ministry of Culture, Sports and Tourism of Republic of Korea: Naju-si, Korea, 2018.

2. Korea Creative Content Agency. 2018 Overseas Content Market Analysis; Ministry of Culture, Sports and Tourism of Republic of Korea: Naju-si, Korea, 2019.

3. Ryu, S.H.; Lee, W.B. Social function of game as a leisure activity. J. Digit. Converg. 2012, 10, 245-251.

4. King, D.L.; Delfabbro, P.H.; Billieux, J.; Potenza, M.N. Problematic online gaming and the COVID-19 pandemic. J. Behav. Addict. 2020, 9, 184-186. [CrossRef]

5. Abel, T.; McQueen, D. The COVID-19 pandemic calls for spatial distancing and social closeness: Not for social distancing! Int. J. Public Health 2020, 65, 231. [CrossRef]

6. Byun, Y.S.; Lee, H.S. Impact of internet addiction on mental health in adolescents. J. Korean Acad. Community Health Nurs. 2007, $18,460-468$.

7. Koepp, M.J.; Gunn, R.N.; Lawrence, A.D.; Cunningham, V.J.; Dagher, A.; Jones, T.; Brooks, D.J.; Bench, C.J.; Grasby, P. Evidence for striatal dopamine release during a video game. Nature 1998, 393, 266-268. [CrossRef] [PubMed]

8. Kwon, S.J.; Kim, K.H.; Lee, H.S. Computer Game Addiction and Physical Health of Korea Children: Mediating Effects of Anxiety. Surv. Res. 2005, 6, 33-50.

9. Lee, Y.K.; Chae, K.M. Relations of computer game addiction and social relationship, adjustment of adolescent. Korean J. Clin. Psychol. 2006, 25, 711-726.

10. Kim, K.W. The study on the internet addiction influencing factor and coping strategies for juvenile. J. Korea Soc. Comput. Inf. 2009, $14,157-165$.

11. Gharib, K.; Homayouni, A.; Yanesari, M.K. P02-90-High levels of stress and addiction to internet. Eur. Psychiatry 2010, 25, 1. [CrossRef] 
12. Hwang, H.; Ryu, S.J. The relationship between internet addiction propensity and psychosocial characteristics of christian college students: Focusing on self-control, self-esteem, self-efficacy, interpersonal efficacy, and loneliness. Korean Assoc. Christ. Couns. Psychol. 2008, 16, 321-348.

13. Park, B.S.; Park, S. Multiple Mediating Effects of Family, Friend and Teacher Relationship on the Relation between Stress and Internet Game Addiction of Adolescents. Health Soc. Welf. Rev. 2016, 36, 61-88.

14. Lee, S.J.; Kim, G.A.; Hong, C.H. The effects of internet use motivation and stress coping on adolescent's pathological internet use: Focused on gender difference. Korean J. Woman Psychol. 2011, 16, 265-284.

15. Lee, H.G. Social and psychological variables predicting violence game addiction of adolescents in the internet. Korean J. Dev. Psychol. 2002, 14, 55-79.

16. Ahn, J.W.; Ku, Y.; Kim, H.C. A novel wearable EEG and ECG recording system for stress assessment. Sensors 2019, 19, 1991. [CrossRef]

17. Correia, B.; Dias, N.; Costa, P.; Pêgo, J.M. Validation of a wireless bluetooth photoplethysmography sensor used on the earlobe for monitoring heart rate variability features during a stress-inducing mental task in healthy individuals. Sensors 2020, $20,3905$. [CrossRef]

18. Cho, D.; Ham, J.; Oh, J.; Park, J.; Kim, S.; Lee, N.-K.; Lee, B. Detection of stress levels from biosignals measured in virtual reality environments using a kernel-based extreme learning machine. Sensors 2017, 17, 2435. [CrossRef]

19. Salai, M.; Vassányi, I.; Kósa, I. Stress detection using low cost heart rate sensors. J. Healthc. Eng. 2016, 2016, 5136705. [CrossRef] [PubMed]

20. Taelman, J.; Vandeput, S.; Vlemincx, E.; Spaepen, A.; Van Huffel, S. Instantaneous changes in heart rate regulation due to mental load in simulated office work. Eur. J. Appl. Physiol. 2011, 111, 1497-1505. [CrossRef]

21. Vuksanović, V.; Gal, V. Heart rate variability in mental stress aloud. Med. Eng. Phys. 2007, 29, 344-349. [CrossRef] [PubMed]

22. Tharion, E.; Parthasarathy, S.; Neelakantan, N. Short-term heart rate variability measures in students during examinations. Natl. Med. J. India 2009, 22, 63-66. [PubMed]

23. Papousek, I.; Nauschnegg, K.; Paechter, M.; Lackner, H.K.; Goswami, N.; Schulter, G. Trait and state positive affect and cardiovascular recovery from experimental academic stress. Biol. Psychol. 2010, 83, 108-115. [CrossRef] [PubMed]

24. Traina, M.; Cataldo, A.; Galullo, F.; Russo, G. Effects of anxiety due to mental stress on heart rate variability in healthy subjects Minerva Psichiatr. 2011, 227, 31.

25. Park, M.C.; Jung, H.C.; Kim, T.S. Design of a stress measurement system for state recognition of game addicts. J. Korea Soc. Comput. Inf. 2017, 22, 87-93.

26. Hafeez, M.; Dawood, I.M.; Kim, J.Y.; Kim, D.J. Study of Game Addiction Pattern by Using Spectral Analysis of EEG. In Proceedings of the Extended Abstracts of HCI Korea, Jeongseon-gun, Korea, 27-29 January 2016; pp. 62-64.

27. Hafeez, M.; Kim, D.J.; Im, S.K.; Kim, J.Y. The Cross Correlation and Power Spectrum Analysis of EEG Attributes between mobile Game Addicts and Non-Addicts. In Proceedings of the HCI Korea, Jeongseon-gun, Korea, 31 January-2 February 2018; pp. 997-999.

28. Kim, D.J.; Kim, H.; Im, S.; Oh, M.S.; Kim, J.; Kim, J.Y. Analysis of EEG Parameters Characteristics for High-Risk Users of Online Games. In Proceedings of the 2019 Spring Conference of ESK, Seogwipo-si, Korea, 15-18 May 2019; p. 237.

29. Meerkerk, G.J.; Van Den Eijnden, R.J.; Vermulst, A.A.; Garretsen, H.F. The compulsive internet use scale (CIUS): Some psychometric properties. Cyberpsychol. Behav. 2009, 12, 1-6. [CrossRef]

30. Young, K.S.; De Abreu, C.N. Internet Addiction: A Handbook and Guide to Evaluation and Treatment; John Wiley \& Sons: Hoboken, NJ, USA, 2010.

31. Littel, M.; Van den Berg, I.; Luijten, M.; van Rooij, A.J.; Keemink, L.; Franken, I.H. Error processing and response inhibition in excessive computer game players: An event-related potential study. Addict. Biol. 2012, 17, 934-947. [CrossRef] [PubMed]

32. Peng, X.; Cui, F.; Wang, T.; Jiao, C. Unconscious processing of facial expressions in individuals with Internet Gaming Disorder. Front. Psychol. 2017, 8, 1059. [CrossRef]

33. Kim, E.J.; Lee, S.Y.; Oh, S.G. The validation of Korean adolescent internet addiction scale (K-AIAS). Korean J. Clin. Psychol. 2003, 22, 125-139.

34. Samaha, A.A.; Fawaz, M.; El Yahfoufi, N.; Gebbawi, M.; Abdallah, H.; Baydoun, S.A.; Ghaddar, A.; Eid, A.H. Assessing the psychometric properties of the internet addiction test (IAT) among Lebanese college students. Front. Public Health $2018,6,365$. [CrossRef]

35. Nam, Y.O. A study on the psychosocial variables of the youth's addiction to internet and cyber sex and their problematic behavior. Korean J. Soc. Welf. 2002, 50, 173-207.

36. Kim, H.; Ha, J.; Chang, W.-D.; Park, W.; Kim, L.; Im, C.-H. Detection of craving for gaming in adolescents with internet gaming disorder using multimodal biosignals. Sensors 2018, 18, 102.

37. Most Popular Core PC Games. Available online: https://newzoo.com/insights/rankings/top-20-core-pc-games (accessed on 10 April 2021).

38. Camm, A.J.; Malik, M.; Bigger, J.T.; Breithardt, G.; Cerutti, S.; Cohen, R.; Coumel, P.; Fallen, E.; Kennedy, H.; Kleiger, R. Heart rate variability: Standards of measurement, physiological interpretation and clinical use. Task Force of the European Society of Cardiology and the North American Society of Pacing and Electrophysiology. Ann. Noninvasive Electrocardiol. 1996, 1, $151-181$.

39. Drew, B.J.; Califf, R.M.; Funk, M.; Kaufman, E.S.; Krucoff, M.W.; Laks, M.M.; Macfarlane, P.W.; Sommargren, C.; Swiryn, S.; Van Hare, G.F. Practice standards for electrocardiographic monitoring in hospital settings: An American Heart Association scientific 
statement from the Councils on Cardiovascular Nursing, Clinical Cardiology, and Cardiovascular Disease in the Young: Endorsed by the International Society of Computerized Electrocardiology and the American Association of Critical-Care Nurses. Circulation 2004, 110, 2721-2746.

40. Hosmer, D.W., Jr.; Lemeshow, S. Applied Logistic Regression, 2nd ed.; John Wiley \& Sons: New York, NY, USA, 2000; pp. 160-164.

41. Klimmt, C.; Hefner, D.; Vorderer, P. The video game experience as "true" identification: A theory of enjoyable alterations of players' self-perception. Commun. Theory 2009, 19, 351-373.

42. Turkay, S.; Kinzer, C.K. The effects of avatar-based customization on player identification. In Gamification: Concepts, Methodologies, Tools, and Applications; IGI Global: Hershey, PA, USA, 2015; pp. 247-272.

43. Bernardi, L.; Wdowczyk-Szulc, J.; Valenti, C.; Castoldi, S.; Passino, C.; Spadacini, G.; Sleight, P. Effects of controlled breathing, mental activity and mental stress with or without verbalization on heart rate variability. J. Am. Coll. Cardiol. 2000, 35, 1462-1469. [CrossRef]

44. Huang, S.; Li, J.; Zhang, P.; Zhang, W. Detection of mental fatigue state with wearable ECG devices. Int. J. Med. Inform. 2018, 119, 39-46. [CrossRef]

45. Jang, E.H.; Kim, A.Y.; Yu, H.Y. Relationships of psychological factors to stress and heart rate variability as stress responses induced by cognitive stressors. Sci. Emot. Sensib. 2018, 21, 71-82. [CrossRef]

46. Lee, J.H.; Yu, J.; Ryu, S.H.; Ha, J.H.; Jeon, H.J.; Park, D.H. Change of heart rate variability in depressive disorder after physical or psychological stress. Sleep Med. Psychophysiol. 2018, 25, 15-20. [CrossRef]

47. Malliani, A.; Pagani, M.; Lombardi, F.; Cerutti, S. Cardiovascular neural regulation explored in the frequency domain. Circulation 1991, 84, 482-492. [CrossRef] [PubMed]

48. Kim, H.G.; Cheon, E.J.; Bai, D.S.; Lee, Y.H.; Koo, B.H. Stress and heart rate variability: A meta-analysis and review of the literature. Psychiatry Investig. 2018, 15, 235. [CrossRef] [PubMed] 